\title{
Investigation of incoherent scatter radar spectra features with stimulated electromagnetic emissions at EISCAT
}

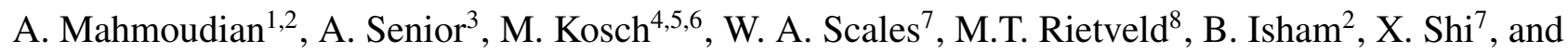 \\ M. Ruohoniemi ${ }^{7}$ \\ ${ }^{1}$ Aerospace Research Institute, Ministry of Science, Research and Technology, Tehran, Iran. \\ ${ }^{2}$ The Inter American University of Puerto Rico, Bayamon, Puerto Rico, USA. \\ ${ }^{3}$ Independent Researcher, Lancaster, UK. \\ ${ }^{4}$ Department of Physics, Lancaster University, Lancaster, UK. \\ ${ }^{5}$ South African National Space Agency (SANSA), Hermanus, South Africa \\ ${ }^{6}$ Dept. of Physics and Astronomy, University of the Western Cape, Bellville, South Africa \\ ${ }^{7}$ Bradely Department of Electerical and Computer Engineering, Virginia Tech. \\ ${ }^{8}$ EISCAT Scientific Association, Ramfjordmoen, Norway
}

\begin{abstract}
The electromagnetic (EM) and electrostatic (ES) emissions can be generated in the ionosphere by high-power highfrequency (HF) radiowaves transmitted from the ground. These emissions known as Stimulated Electromagnetic Emissions (SEE) can be employed for remote measurment of ionospheric parameters. The experimental data from recent HF heating experiment near fourth electron gyro-frequency $4 \mathrm{f}_{c e}$ at EISCAT are presented. This paper compares the temporal behavior

5 of Wideband SEE (WSEE) within 1-50 kHz of the pump frequency and Narrowband SEE (NSEE) within a few Hertz to $100 \mathrm{~Hz}$ of the tranmission fequency to the time evolution of enhanced ion and plasma lines in the incoherent scatter radar (ISR) spectrum. The correlation of WSEE feature such as downshifted maximum (DM), upshifted maximum (UM), and broad upshifted maximum (BUM), with HF enhances ion line (HFIL) is shown. It has been shown that WSEE spectral lines can be used to reproduce the HFIL including altitude range, rise and decay time, maximum and minimum amplitude. The electron desnity profile near the interaction altitude, magnetic field strength $B_{0}$ as well as altitude profile of the EHIL can be estimated using the temporal evolution of WSEE features near $\mathrm{n} f_{c e}$.
\end{abstract}

\section{Introduction}

The field of active space experiments using high-power radiowave transmission from the ground and detection of the secondary electromagnetic waves excited in the ionosphere as a result of interaction with the local plasma has been growing rapidly over the past decade. The detected sideband on the ground associated with the reradiated signal in the ionosphere, known as Stimulated Electromagnetic Emission (SEE), has a footprint of the generated electromagnetic (EM) and electrostatic (ES) waves. The measured SEE spectrum can be used to diagnose ionospheric parameters. Combination of SEE measurement and other remote sensing tools can lead to diagnostic information about the plasma instabilities and processes in the heated ionospheric region. 
The main Wideband SEE features appear in the frequency band of 1 to $100 \mathrm{kHz}$ and include the Upshifted Maximum (UM) $\Delta f \approx 9 \mathrm{kHz}$, Downsifted Maximum (DM) $\Delta f \approx 8.5-11 \mathrm{kHz}$, Broad Upshifted Maximum (BUM) $\Delta f \approx 15-100 \mathrm{kHz}$, and Downshifted Peak (DP) $\Delta f \approx 1-2 \mathrm{kHz}$. There are several other WSEE features such as Narrow Continium (NC), fast narrow continuum (FNC), and broad continuum (BDE), which are not used in this study. The initial parametric decay of an EB wave

5 into another EB wave and a nearly perpendicularly propagating IA (or electrostatic ion cyclotron) wave has been implemented to interpret the DP, nDP, and UP features (Huang and Kuo, 1995). The decaying EB wave has the frequency $f_{0}$ and is produced through a thermal OTSI in which the small-scale FAS is the decay mode. The DM would be suppressed at both $f_{0} \approx n f_{c e}$ and $f_{0} \approx n f_{c e}+f_{l h}$, where $f_{l h}$ is the lower-hybrid frequency. The UH resonance region, where the pump frequency $f_{0}$ is equal to $\sqrt{\omega_{p e}^{2}+\Omega_{c e}^{2}}$, is typically a few kilometers below the pump reflection height. The suppression of the DM for $f_{0}$ near $s f_{c e}$ has also been attributed to the mode conversion of the propagating UH waves to resonantly excited non-propagating EB waves (Rao ad Kaup, 1990, 1992). Narrowband SEE feature (NSEE) within $1 \mathrm{kHz}$ of the pump frequency have been discovered very recently. The NSEE features such as Magnetized Stimulated Brillouin Scatter (MSBS) and Stimulated Ion Bernstien Scatter (SIBS) were observed for the first time after recent update of the HF facility at HAARP in 2007, which is now the most powerful HF transmitter in the world. Ion gyro structures associated with SIBS process and ordered by Oxygen ion gyro-frequency of the order of $50 \mathrm{~Hz}$, and Hydrogen ion gyro-frequency of the order of $800 \mathrm{~Hz}$ during active geomagnetic conditions have been reported during HF pump heating of the ionosphere at HAARP (Fu et al., 2013; Mahmoudian et al., 2013; Bordikar et al., 2014a, b).

The main focus of this paper is to use the BUM, DM, and UM features to investigate the measured parameters using incoherent scatter radar (ISR). The main goal of the present work is to develop a sustainable remote sensing technique using ground SEE observations in order to replace the ISR measurements. The main ISR measurements are enhanced ion lines (EHIL) and enhanced plasma line (EHPL). The EHPL feature in the ISR spectrum corresponds to the excited Langmuir waves. The ion acoustic waves excited as a result of pump wave heating appear in the EHIL feature. It should be noted that natural IA waves present in the ionosphere but heavily damped. As the electron temperature increases during radiowave heating, the IA wave grows faster and to larger amplitudes.

ISR PL are less investigated in comparision ion line spectra, which is mainly due to low strength and nstrumentally challenging to detect, as well as the broadband nature of PL. The ISR IL measurements are sensitive to $\mathrm{N}_{e}, \mathrm{~T}_{e}, \mathrm{~T}_{i}, \mathrm{~V}_{\text {los }}, \nu_{\text {in }}$, and $\mathrm{N}_{i} / \mathrm{N}_{e}$. The PL measurement can lead to sensitive measurement of $\mathrm{N}_{e}$ in the daytime (Isham et al., 2000). The ISR data provides the ability to extract wind and waves with high temporal and altitudinal resolution, much like lidars. While ISR measurements are very accurate and comprehensive, the lack of availability, large size, and the maintanance cost limit the application of ISR measurements. Therefore, we investiagte the potential of the ground-based SEE measurements as a replacement/complement to the ISR diagnostics.

This paper is organized as follow. The first section explains the experiment set up conducted at the EISCAT facility on Feburary 22 and 23, 2012. The experimental observations are described in section two. The discussion section elaborates the similarities between the SEE and ISR measurements and the self-consistency of SEE in monitoring the current state of the ionosphere. The conclusion and summary is provided. 


\section{Experiment setup}

The SEE receiver was located at $69.64 \mathrm{~N}, 19.49 \mathrm{E}$, approximately $13 \mathrm{~km}$ ENE of the HF facility. The antenna was a broadband resistively-loaded folded dipole. The RF signal from the antenna was sampled at $50 \mathrm{MHz}$ and downsampled to a rate of 6.25 $\mathrm{MHz}$ for storage and later analysis.

Between 15:00 and 17:00 UT on 2012-02-22, the pump beam was O-mode polarised and pointed field-aligned (12 degrees south of zenith). The pump was cycled 18 minutes on, 12 minutes off. During each pump-on period, the pump frequency was held at exactly $5.3 \mathrm{MHz}$ for $130 \mathrm{~s}$ and then stepped upwards in frequency by $3.125 \mathrm{kHz}$ every $10 \mathrm{~s}$, thus passing through the fourth gyroharmonic. The EISCAT ISR was used throughout this study to monitor the time evolution of enhanced-ion-lines (EHIL) as well as the variation in altitudes of the excited irregularities. The main purpose of this study is to compare the ISR observations with the SEE feature in order to develop sustainable remote sensing technique using SEE measurements and replace the ISR observations. A similar experiment was conducted on Fuburary 23, 2012 from 15:30 to 18:00 UT, and the corresponding data is shown in Figures 7 and 8.

\section{Experimental observations}

Several instruments are used in order to investigate the ISR spectral features including EHIL and EHPL with NSEE and WSEE observations. Figure 1 shows the ionogram data at the begining of each heating cycle 15:00 UT, 15:30 UT, and 16:00 UT. As can be seen, the maximum electron plasma frequency $\omega_{p e}$ is $5.8 \mathrm{MHz}, 5.7 \mathrm{MHz}$, and $5.2 \mathrm{MHz}$, correspondig to the heating cycles 1,2 , and 3 . The interaction region for case 3 is very limited as the maximum plasma frequency drops to $5.2 \mathrm{MHz}$ during this experiment. Considering the frequency sweep from $5.3 \mathrm{MHz}$ to $\sim 5.6 \mathrm{MHz}$ during the experiments, the altitude range of interaction varies from $15 \mathrm{~km}$ to $30 \mathrm{~km}$ between cases 1 and 2 , respectively. This is a unique feature in the ionogram data that determines the shape of WSEE and the time period for the pump frequency sweep through $\mathrm{n} f_{c e}$. Therefore, the behavior of WSEE features such as DM and the time period for the disappearance and reappearance of WSEE spectral lines can be used to estimate the scale-Height, magnetic field strength $B_{0}$, and altitude profile of electron density in the ionosphere.

The spectrum of WSEE features in the frequency range of $-40 \mathrm{kHz}$ to $+80 \mathrm{kHz}$ including DM, UM, BUM, and DP are shown in Figure 2. The variation of WSEE strength, which corresponds to the interaction with the plasma layer shows a much stronger interaction in case 1 and 2. A comparision with the electron density profiles in Figure 1 also validates the observations. Time variation of WSEE features associated with Figure 2 is shown in Figure 3. The behavior of EHIL in ISR spectra is shown in Figure 4 . The enhanced electron temperature is suppressed substantially as the pump frequency approaches $4 f_{c e}$. The behavior of increased $\mathrm{T}_{e}$ shows a very similar trend to the EHIL as well as strength of DM. The experimental observations associated with a similar experimental set up conducted on Fuburary 23, 2012 are presented in Figure 7 and 8. A plot corresponding to the narrowband SEE (NSEE) features within $100 \mathrm{~Hz}$ of the pump frequency is included in Figure 8. The spectral lines related to the Magnetized Stimulated Brillouin Scatter (MSBS) are observed that could explain the different behavior of the ISR EHIL and WSEE features for the conducted experiment on Feburary 23, 2012. 

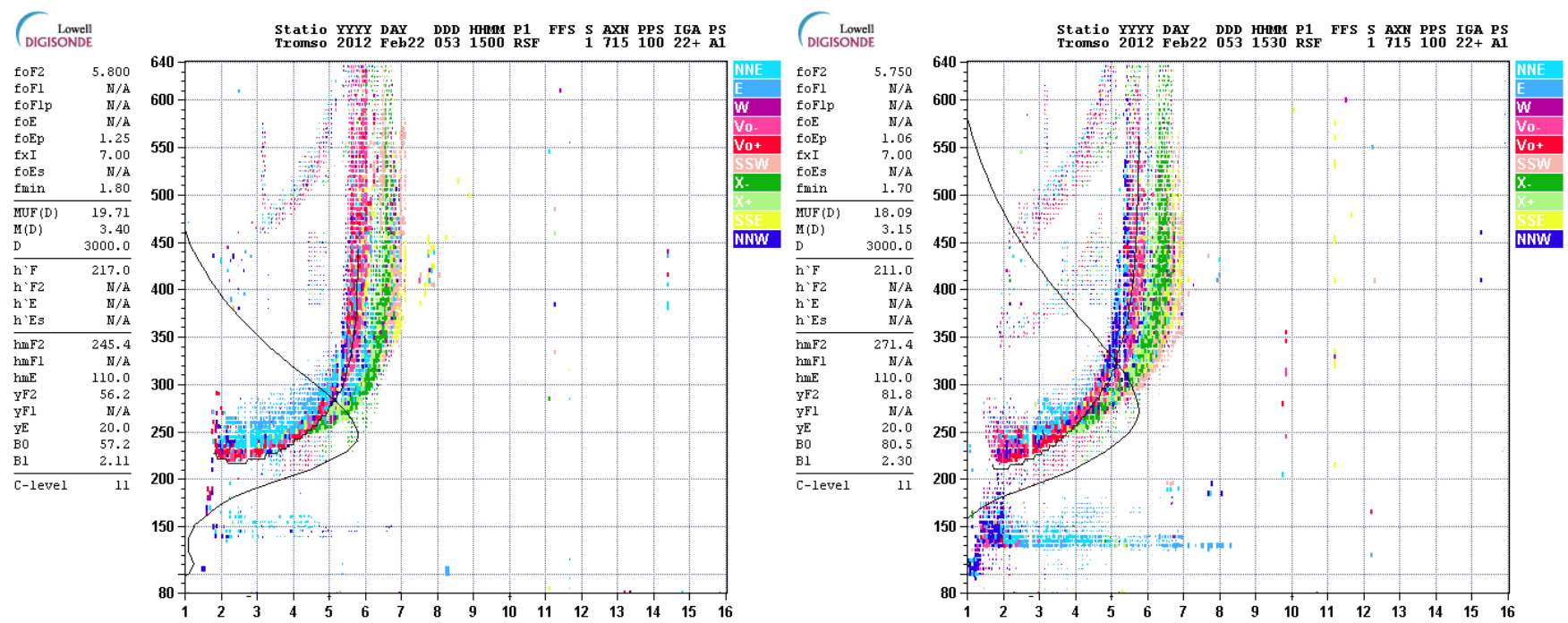

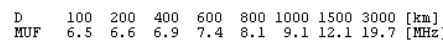

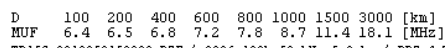

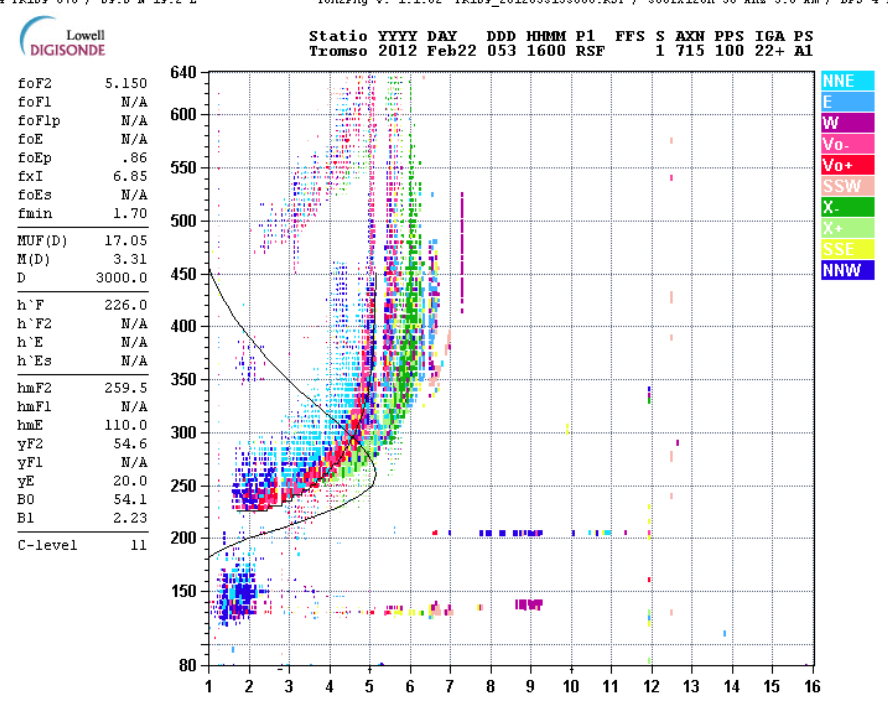

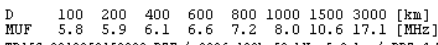

Figure 1. Ionogram data plots associated with heating cycles 1, 2, and 3 starting at 15:00 UT, 15:30 UT, and 16:00 UT, respectively.

\section{Discussion}

The weak dependence of DM on $f_{0}$ is attributed to the $\mathrm{LH}$ (lower-hybrid) waves present in this parametric instability. The spectral widening of the DM above $\mathrm{n} f_{c e}$ can be explained by the thermal dispersion branch of the LH mode. This is due to the effectively unmagnetized ions for frequencies well above the ion gyro-frequency $\left(\Omega_{c i}\right)$ whereas the electrons are magnetized. The 5 broad upshifted maximum (BUM) feature in the SEE spectrum should occur several kilometers below the double-resonance al- 
titude. The double resonance layer occurs when the pump frequency $f_{0}$ equals $n f_{c e}\left(h_{d}\right)$ and $f_{U H}\left(h_{d}\right)\left(=\sqrt{\omega_{p e}^{2}+\Omega_{c e}^{2}}\right)$, where $h_{d}$ denotes the double resonance layer.

The generation process of DP is attributed to the parametric decay of an EB wave into another EB wave and a nearly perpendicularly propagating IA (Huang and Kuo, 1995; Leyser, 2001). A close comparison of the IA spectral line (NSEE feature) produced through the MSBS process and the DP emission line which corresponds to the IA line propagating near the perpendicular direction, shows a clear anti-correlated behavior. This could be related to the propagation characteristic of the IA waves excited through these two processes.

The empirical relation for the peak frequency of the BUM spectral line derived by Grach is

$$
f_{B U M}-f_{1}=f_{1}-n f_{c e}\left(h_{d}\right) \quad f_{1}=f_{0}-\delta f / 2
$$

10 where $f_{B U M}$ is the BUM feature frequency, $f_{0}$ is the pump frequency, $\delta f / 2$ is approximately equal to $\delta f_{D M} \approx f_{L H} . f_{L H}$ is the lower-hybrid frequency.

The characteristics of the two processes are compared with the present experimental observations as follows:

As can be seen in Figure 3 and for the DM spectral line, the initial overshoot in the signal strength was followed by a decay and thereafter an increase back to a high level after pump-on is observed. The time-scale of the temporal evolution in WSEE features and HFIL are consistent and show a very similar trend. The variation of HFIL amplitude shows a strong correlation with the DM spectral line. We have shown that a close comparison of DM and HFIL amplitude throughout the heating cycle as the pump frequency passes through $4 f_{c e}$, can be used to estimate the electron density profile, and reproduce the altitude-time profile of plasma lines in ISR spectrum. The comparison over several cycles reveals a threshold level for the HFIL to appear in the ISR spectrum. The initial rise time correlates well with the enhanced electron temperature.

The general data reduction technique used in this study is shown in Figure 5. According to this figure, the information on the BUM appearance in the SEE spectra can be used to determine the exact value of $4 f_{c e}$, magnetic field strength, an estimated altitude of the double resonance layer, and $n_{e}$ at this altitude. The value of $4 f_{c e}$ will be checked with other WSEE features such as DM. It may be noted that the BUM is excited also for $f_{0}$ slightly below $s f_{c e}$, down to $f_{0} \approx s f_{c e}-10 \mathrm{kHz}(s=5)$ (Frolov et al., 1996; 2000). The BUM intensity is maximum usually for $f_{B U M} 30-40 \mathrm{kHz}$, which is consistent with the experimental results presented in Figure $3(\sim 40 \mathrm{kHz})$.

Considering the required condition on $f_{0}$ proximity to $n f_{c e}$, the BUM appearance occurs when $f_{0} \sim n f_{c e}-10-15 \mathrm{kHz}$. The BUM spectral emission line in case 1, appears at $t=575 \mathrm{~s}$ from the beginning of the heating cycle (15:09 UT), which corresponds to the pump frequency of $\sim 5.34 \mathrm{MHz}$. The BUM disappearance from the WSEE spectrum occurs at the same time that the DM emission line reaches its maximum amplitude, which is at $t \approx 15: 15 \mathrm{UT}$. For the first heating cycle on 30 February 22, 2012, this condition is satisfied with the pump frequency 5.433 MHz at $t=15: 09: 58 \mathrm{UT}$. According to the double resonance condition, this frequency is equivalent to the $f_{0}=n f_{c e}=\sqrt{\Omega_{c e}^{2}+\omega_{p e}^{2}}$. Therefore, the variation of $f_{B U M}$ is a direct manifestation of the change in the interaction altitude. This behavior can be implemented in order to reconstruct the ISR EHIL spectra. According to Figure 2a and 3a, the BUM spectral line appears at 15:09 UT which corresponds to the 
pump frequency of $5.435 \mathrm{MHz}$. Considering the above mentioned condition on the pump frequency for the excitation of BUM, $f_{0} \approx n f_{c e}-\sim 10 \mathrm{kHz}, 4 f_{c e} \approx 5.445 \mathrm{MHz}$ during this experiment. The estimated magnetic field strength is included in Table 1 using the IGRF (International Geomagnetic Reference Field) model and in the altitude range of $200 \mathrm{~km}$ to $280 \mathrm{~km}$ with 5 $\mathrm{km}$ steps. According to Table 1, the measured $4 f_{c e}$ equivalent to the altitude of $\sim 233 \mathrm{~km}$ and the magnetic field strength of

$57.7398 \mathrm{e}-06 \mathrm{nT}$ (corresponding to $f_{c e} \sim 1.3613 \mathrm{MHz}$ ). A close agreement between the time evolution of the WSEE spectral lines and EHIL can be used to predict the time-altitude profile of this ISR feature. The maximum amplitude of the DM spectral line and disappearance of the BUM feature can be implemented as a marker for the time and altitude at which the EHIL will recover to its initial amplitude. The formation of BUM feature coincidences with the minimum amplitude of DM spectral line, which is consistent with the behavior of EHIL observed by ISR. Therefore, while the timing of the events including the minimum and maximum altitude of the EHIL matches well with the minimum and maximum amplitude of DM spectral line, the altitude range of EHIL variation can be estimated with the reverse behavior. The time at which the DM emission line reaches its maximum amplitude shows a good agreement with the recovery phase of EHIL versus altitude. As a result, this parameter can be used to determine the range of altitude variation associated with EHIL. According to Figure 3a, the recovery time of the DM emission line is about 6 min and the pump frequency variation is of the order of $109 \mathrm{kHz}$. In other words, the time of minimum amplitude of the DM feature (appearance of BUM) can be used to determine the maximum altitude of EHIL. The maximum altitude estimated from the WSEE feature is $233 \mathrm{~km}$, which is consistent with the EHIL observed by ISR. The estimated electron density from the matching condition $(\sim 5.3 \mathrm{MHz})$ which is in agreement with the ionogram data presented in Figure 1a. Knowing the typical scale-height of the ionosphere, the altitude profile of the electron density can be estimated using this technique. The time of DM recovery to the initial amplitude (BUM disappearance) is 15:15 UT, which corresponds to the $5.539 \mathrm{MHz}$. According to Table 1, this frequency is equivalent to the altitude $\sim 205 \mathrm{~km}$, which is similar to the minimum altitude of EHIL shown in Figure 4b. The similar calculations for the second and third heating cycles also show a similar result with the EHIL observations.

Also the measured enhanced electron temperature $T_{e}$ (Figure $7 \mathrm{a}$ ) as well as the comparison between the EHIL versus altitude profile (Figure 7b) with the NSEE spectrum shown in Figure 8b shows that for the heating cycle between 17:00 UT and 17:30 UT a strong correlation occurs about $6 \mathrm{~min}$ into the heating cycle. As the electron temperature increases dramatically $T_{e}$ and the EHIL does not show a typical behavior but rather a chaotic increase over the altitude range of interaction, the DM, and DP emission lines are weakened in the WSEE spectrum. A very strong NSEE feature observed associated with this heating cycle. The correlation between these two processes can be clearly seen in Figures 8a and 8b. The NSEE feature observed with a frequency offset of $\sim 55 \mathrm{~Hz}$. This emission line is attributed to the Electrostatic Ion Cyclotron (EIC) waves excited through the MSBS process (Bernhardt et al., 2010; Mahmoudian et al., 2014a, b). This behavior shows that the temporal evolution of EHIL in ISR spectrum can be predicted by the NSEE and WSEE observations. A close comparision between the stength of DM line in time (Figure 2) and the enhanced $T_{e}$ over the three heating cycles (Figure 4a) reveals a strong correlation. More advanced computational modeling is required to complete the remote sensing of ionospheric parameters with SEE observations and replacement of ISR measurements. 


\begin{tabular}{|c|c|c|c|c|c|c|c|c|}
\hline $200 \mathrm{~km}$ & $205 \mathrm{~km}$ & $210 \mathrm{~km}$ & $215 \mathrm{~km}$ & $220 \mathrm{~km}$ & $225 \mathrm{~km}$ & $230 \mathrm{~km}$ & $\begin{array}{c}235 \mathrm{~km} \\
240 \mathrm{~km}\end{array}$ \\
$5.9942 \mathrm{MHz}$ & $5.5073 \mathrm{MHz}$ & $5.4960 \mathrm{MHz}$ & $5.4847 \mathrm{MHz}$ & $5.4735 \mathrm{MHz}$ & $5.4623 \mathrm{MHz}$ & $5.4511 \mathrm{MHz}$ & $5.4400 \mathrm{MHz}$ & $5.4289 \mathrm{M}$ \\
\hline $245 \mathrm{~km}$ & $250 \mathrm{~km}$ & $255 \mathrm{~km}$ & $260 \mathrm{~km}$ & $265 \mathrm{~km}$ & $270 \mathrm{~km}$ & $275 \mathrm{~km}$ & $280 \mathrm{~km}$ & \\
$5.4178 \mathrm{MHz}$ & $5.4067 \mathrm{MHz}$ & $5.3956 \mathrm{MHz}$ & $5.3846 \mathrm{MHz}$ & $5.3736 \mathrm{MHz}$ & $5.3627 \mathrm{MHz}$ & $5.3518 \mathrm{MHz}$ & $5.3408 \mathrm{MHz}$ & \\
\hline
\end{tabular}

\section{Conclusions}

This paper presents the first study on the time evolution of the SEE features during pump frequency variation near the harmon5 ics of the electron gyro-frequency $n f_{c e}$ to remotely measure ionospheric parameters. The main WSEE features used in this study to make a comparison with the incoherent scatter radar (ISR) observations are the downshifted maximum (DM), broad upshifted maximum (BUM), and upshifted maximum (UM). The distinct behavior of SEE spectra during this experimental set up provides a unique opportunity to reproduce the measurements by incoherent scatter radar. As the pump frequency approaches $n f_{c e}$, the DM spectral line becomes stronger initially, then decays to a minimum amplitude as the $f_{0}$ gets closer to the gyro-frequency. A recovery phase to the amplitudes close to the initial condition is observed. The BUM spectral line develops at the same time as the DM reaches a minimum amplitude and disappears from the WSEE spectrum at the same time the DM recovers to its initial amplitude. This time evolution represents a very similar trend to the variation of enhanced-ion-line (EHIL) in the ISR spectra, which shows an initial enhancement, then a drop in altitude, followed by a recovery phase. It has been shown that a data reduction technique can be developed to determine the electron density profile, electron gyro-frequency variation, as well as the complete behavior of EHIL in ISR spectra including altitude variation, can completely be reproduced using this technique. This characteristic is used to reproduce the ISR measurements with WSEE features.

A close comparison of the measured enhanced electron temperature using ISR, shows a close agreement with the time variation of the DM spectral line as the pump frequency varies throughout the heating cycle. A more detailed study including the numerical simulations of the DM feature in order to obtain the relationship between the strength of the WSEE and enhanced $T_{e}$ could result in a strong remote sensing technique. This will be the subject of the future investigations.

Acknowledgements. This work has been supported in part by National Science Foundation (NSF). The data presented in this paper can be downloaded from the following links. 


\section{References}

Bernhardt, P. A., C. A. Selcher, R. H. Lehmberg, S. Rodriguez, J. Thomason, M. McCarrick, and G. Frazer (2009), Determination of the electron temperature in the modified ionosphere over HAARP using the HF pumped Stimulated Brillouin Scatter (SBS) emission lines, Ann. Geophys., 27, 4409-4427, doi:10.5194/angeo-27-4409-2009.

Bernhardt, P. A., C. A. Selcher, R. H. Lehmberg, S. P. Rodriguez, J. F. Thomason, K. M. Groves, M. J. McCarrick, and G. J. Frazer (2010), Stimulated Brillouin scatter in a magnetized ionospheric plasma, Phys. Rev. Lett., 104, 165,004, doi:10.1103/PhysRevLett.104.165004.

Bordikar, M. R., W. A. Scales, A. Samimi, P. A. Bernhardt, S. Briczinski, and M. J. McCarrick (2013), First observations of minority ion $\left(\mathrm{H}^{+}\right)$structuring in stimulated radiation during second electron gyro-harmonic heating experiments, Geophys. Res. Lett., 40, 1479-1483, doi:10.1002/grl.50327.

Bordikar, M. R., W. A. Scales, A. Mahmoudian, H. Kim, P. A. Bernhardt, R. Redmon, A. R. Samimi, S. Brizcinski, and M. J. McCarrick (2014), Impact of active geomagnetic conditions on stimulated radiation during ionospheric second electron gyroharmonic heating, J. Geophys. Res. Space Physics, 119, doi:10.1002/2013JA019367.

Djuth F. T., P. A. Bernhardt, L. Zhang, Magnetized Stimulated Brillioun Scatter Excited in the F-region and sporadic-E at Arecibo Observatory, Triennial Earth-Sun Summit 20-24 May 2018.

Fu, H., W. A. Scales, P. A. Bernhardt, A. Samimi, A. Mahmoudian, S. J. Briczinski, and M. J. McCarrick (2013), Stimulated Brillouin scatter and stimulated ion Bernstein scatter during electron gyroharmonic heating experiments, Radio Sci., 48, 607-616, doi:10.1002/2013RS005262.

Frolov, V. L., S. M. Grach, L. M. Erukhimov, G. P. Komrakov, E. N. Sergeev, B. Thidd, and T. Carozzi, Peculiarities in the evolution of the BUM of stimulated radio emission of the ionosphere, Radiophys. Quantum Electron., Engl. Transl., 39, 241-254, 1996.

Frolov, V. L., E. N. Ermakova, L. M. Kagan, G. P. Komrakov, E. N. Sergeev, and P. Stubbe, Features of the broad upshifted structure in stimulated electromagnetic emission spectra, J. Geophys. Res., 105, 20919-20933, 2000

Grach, S., S. Sergeev, V. Yashnov, and V. Kotov (2008), Spectra of stimulated electromagnetic emission of the ionosphere during pump frequency sweeping near gyroharmonics. II. Discussion of the results, Radiophys. Quantum Electron., 51, 499-514.

Huang, J., and S. P. Kuo (1995), A generation mechanism for the downshifted peak in stimulated electromagnetic emission spectrum, J. Geophys. Res., 100(A11), 21433-21438, doi:10.1029/95JA02302.

Isham, B., C. A. Tepley, M. P. Sulzer, Q. H. Zhou, M. C. Kelley, J. S. Friedman, and S. A. González (2000), Upper atmospheric observations at the Arecibo Observatory: Examples obtained using new capabilities, J. Geophys. Res., 105(A8), 18609-18637, doi: 10.1029/1999JA900315.

Leyser, T. B. (2001), Stimulated electromagnetic emissions by high-frequency electromagnetic pumping of the ionospheric plasma, Space Sci. Rev., 98, 223-328.

Mahmoudian, A., W. A. Scales, P. A. Bernhardt, A. Samimi, E. Kendall, J. M. Ruohoniemi, B. Isham, O. Vega-Cancel, and M. Bordikar (2013a), Ion gyro-harmonic structuring in the stimulated radiation spectrum and optical emissions during electron gyro-harmonic heating, J. Geophys. Res. Space Physics, 118, 1270-1287, doi:10.1002/jgra.50167.

Mahmoudian, A., W. A. Scales, P. A. Bernhardt, H. Fu, S. J. Briczinski, and M. J. McCarrick (2013b), Investigation of ionospheric stimulated Brillouin scatter generated at pump frequencies near electron gyroharmonics, Radio Sci., 48, 685-697, doi:10.1002/2013RS005189.

Mahmoudian, A., W. A. Scales, P. A. Bernhardt, B. Isham, E. Kendall, S. J. Briczinski, N. E. B. Fuentes, and O. Vega-Cancel (2014a), Electron gyroharmonic effects on ionospheric stimulated Brillouin scatter, Geophys. Res. Lett., 41, 5710-5716, doi:10.1002/2014GL061050. 
Mahmoudian, A., W. A. Scales, M. R. Bordikar, A. Samimi, and P. A. Bernhardt (2014b), Narrowband Stimulated Electromagnetic Emissions (SEE) Spectra: A New Ionospheric Diagnostic Technique, General Assembly and Scientific Symposium, URSI.

5 Mahmoudian, A., W.A. Scales, B.J. Watkins, P.A. Bernhardt, B. Isham, O. Vega-Cancel, J.M. Ruohoniemi (2016), Investigation of third gyro-harmonic heating at HAARP using stimulated radio emissions and the MUIR and Kodiak radars, Advances in Space Research, Vol 59, Issue 1, Pages 337-350, http://dx.doi.org/10.1016/j.asr.2016.09.029.

Mishin, E., B. Watkins, N. Lehtinen, B. Eliasson, T. Pedersen, and S. Grach (2016), Artificial ionospheric layers driven by high-frequency radiowaves: An assessment, J. Geophys. Res. Space Physics, 121, 3497-3524, doi:10.1002/2015JA021823.

10 Norin, L., T. B. Leyser, E. Nordblad, B. Thide, and M. McCarrick (2009), Unprecedentedly strong and narrow electromagnetic emissions stimulated by high frequency radio waves in the ionosphere, Phys. Rev. Lett., 065003, doi:10.1103/PhysRevLett.102.065003.

Rao, N. N., and D. J. Kaup (1990), Upper hybrid mode conversion and resonance excitation of Bernstein modes in ionospheric heating experiments, J. Geophys. Res., 95(A10), 17245-17252, doi: 10.1029/JA095iA10p17245.

Rao, N. N., and D. J. Kaup (1992), Excitation of electron cyclotron harmonic waves in Ionospheric Modification Experiments, J. Geophys. Res., 97(A5), 6323-6341, doi: 10.1029/91JA02437. 

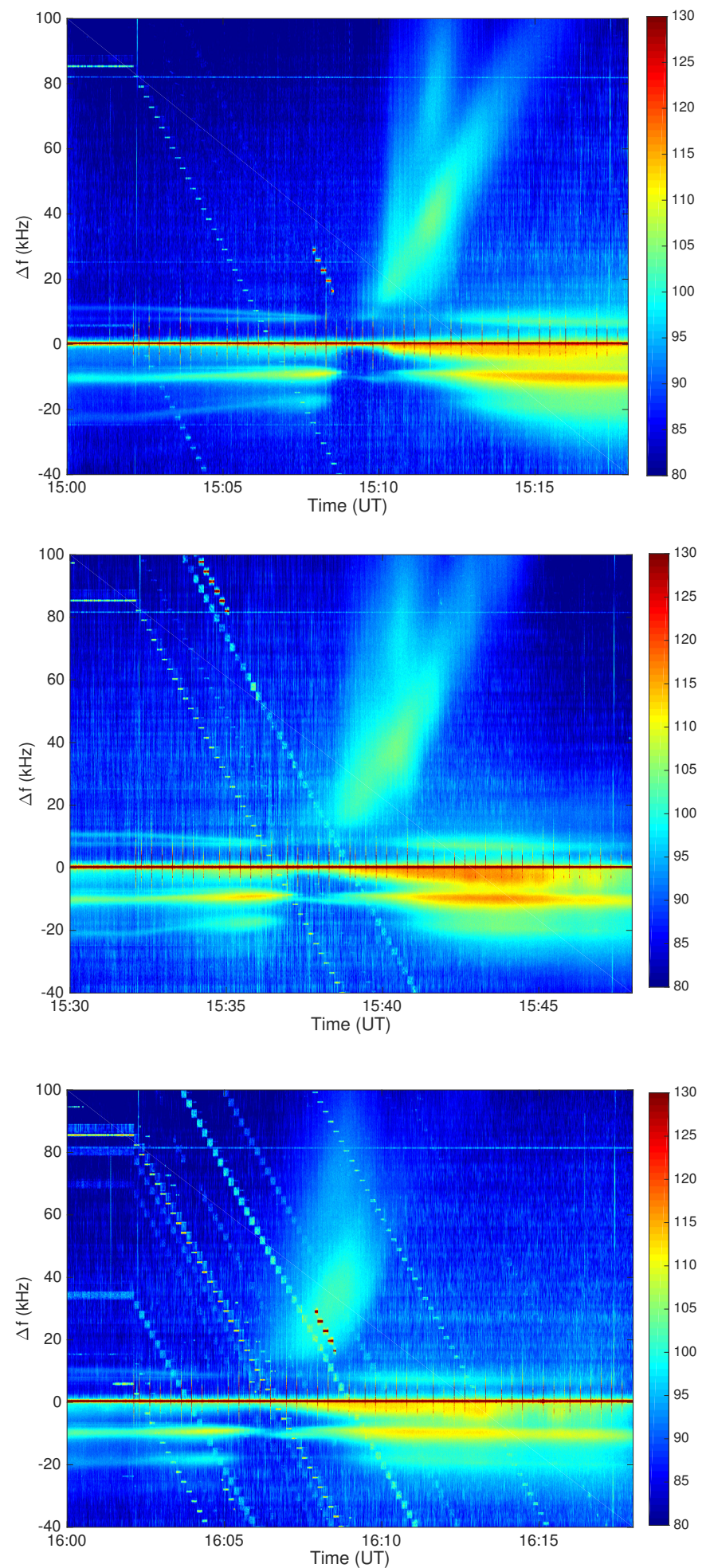

Figure 2. Wideband SEE (WSEE) features including DM, DP, BUM, and UM in the frequency range of $-40 \mathrm{kHz}$ to $+100 \mathrm{kHz}$ of the pump frequency. 

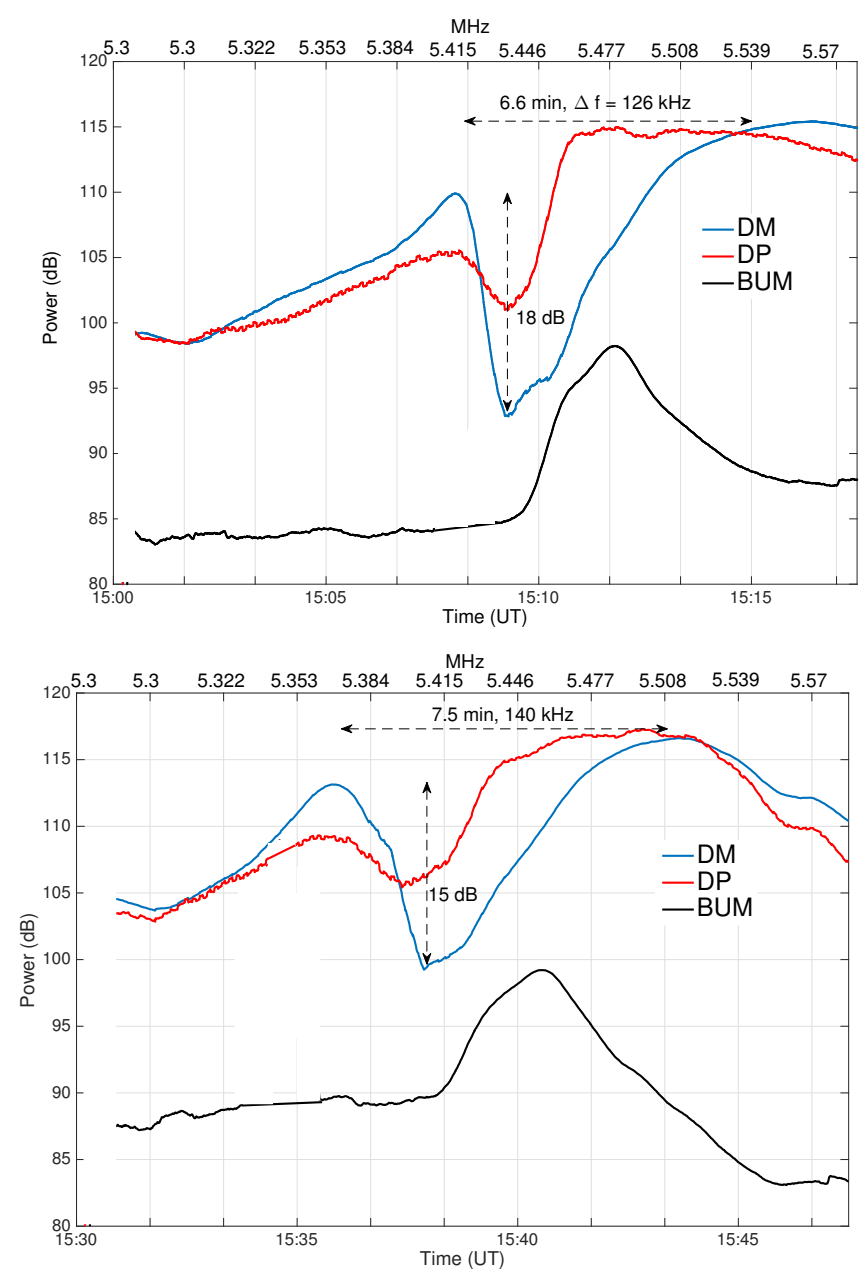

$\mathrm{MHz}$

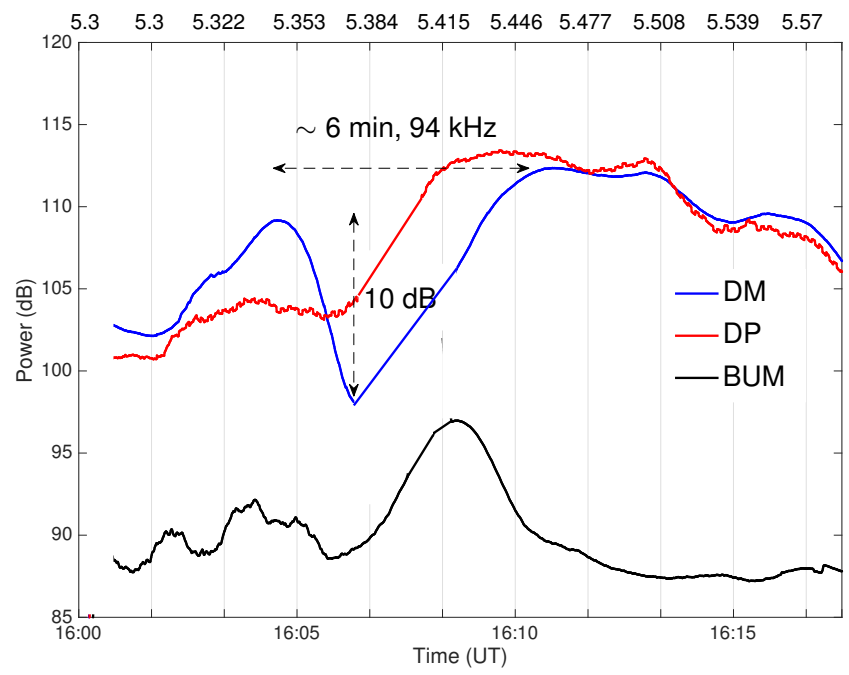

Figure 3. Time variation of WSEE features associated with Figure 2. 


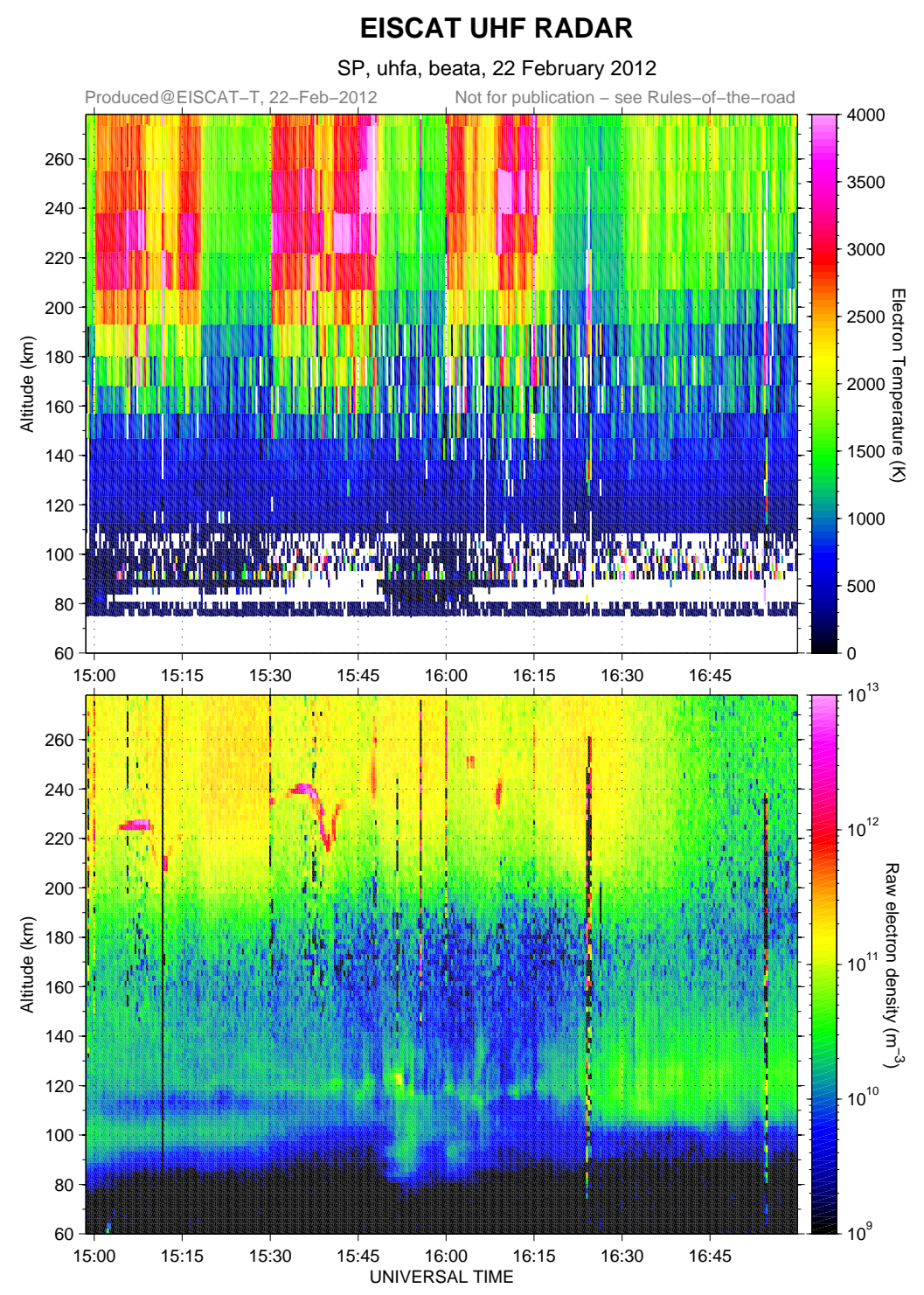

Figure 4. Enhanced electron temperature and enhanced ion lines (EHIL) corresponding to three heating cycles. 


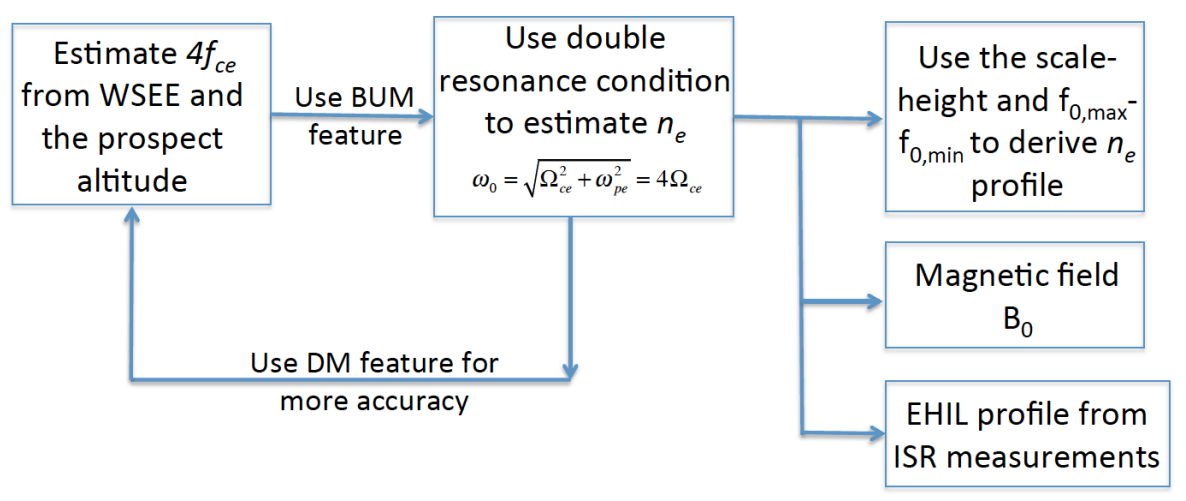

Figure 5. Data reduction technique using WSEE features in order to obtain estimate magnetic field strength, electron density profile, and reproduce EHIL versus altitude profile.

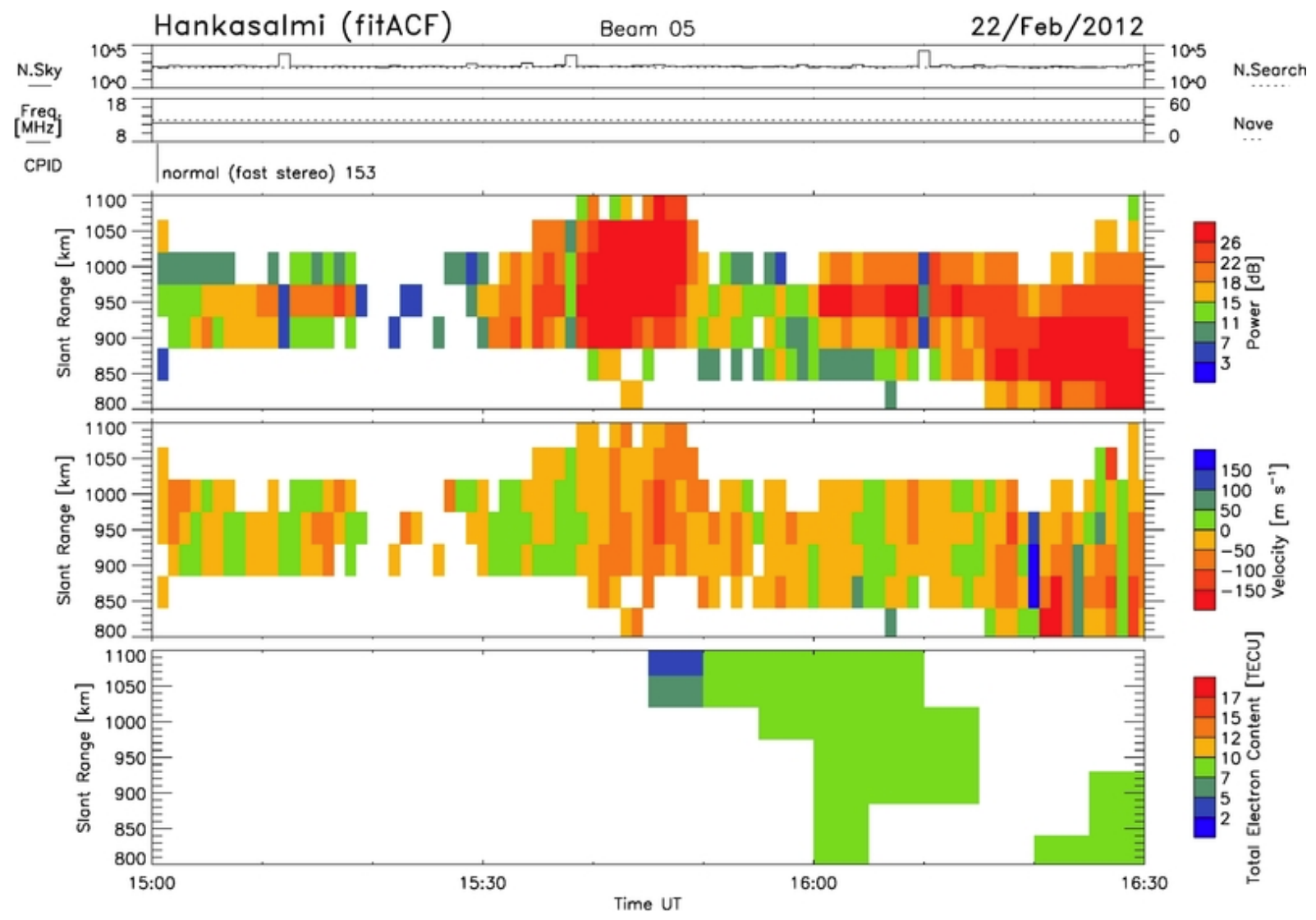

Figure 6. TEXT 


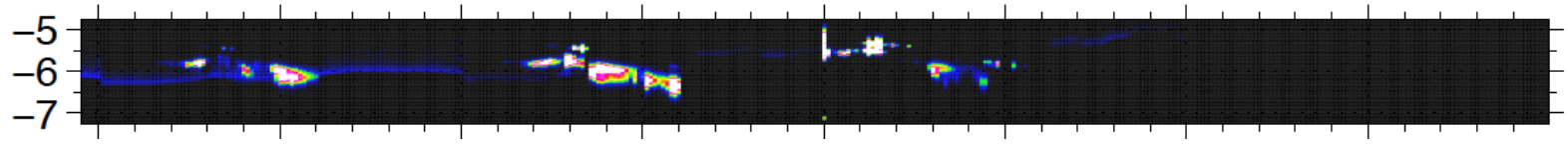

Figure 7. TEXT 


\section{EISCAT UHF RADAR}

SP, uhfa, beata, 23 February 2012

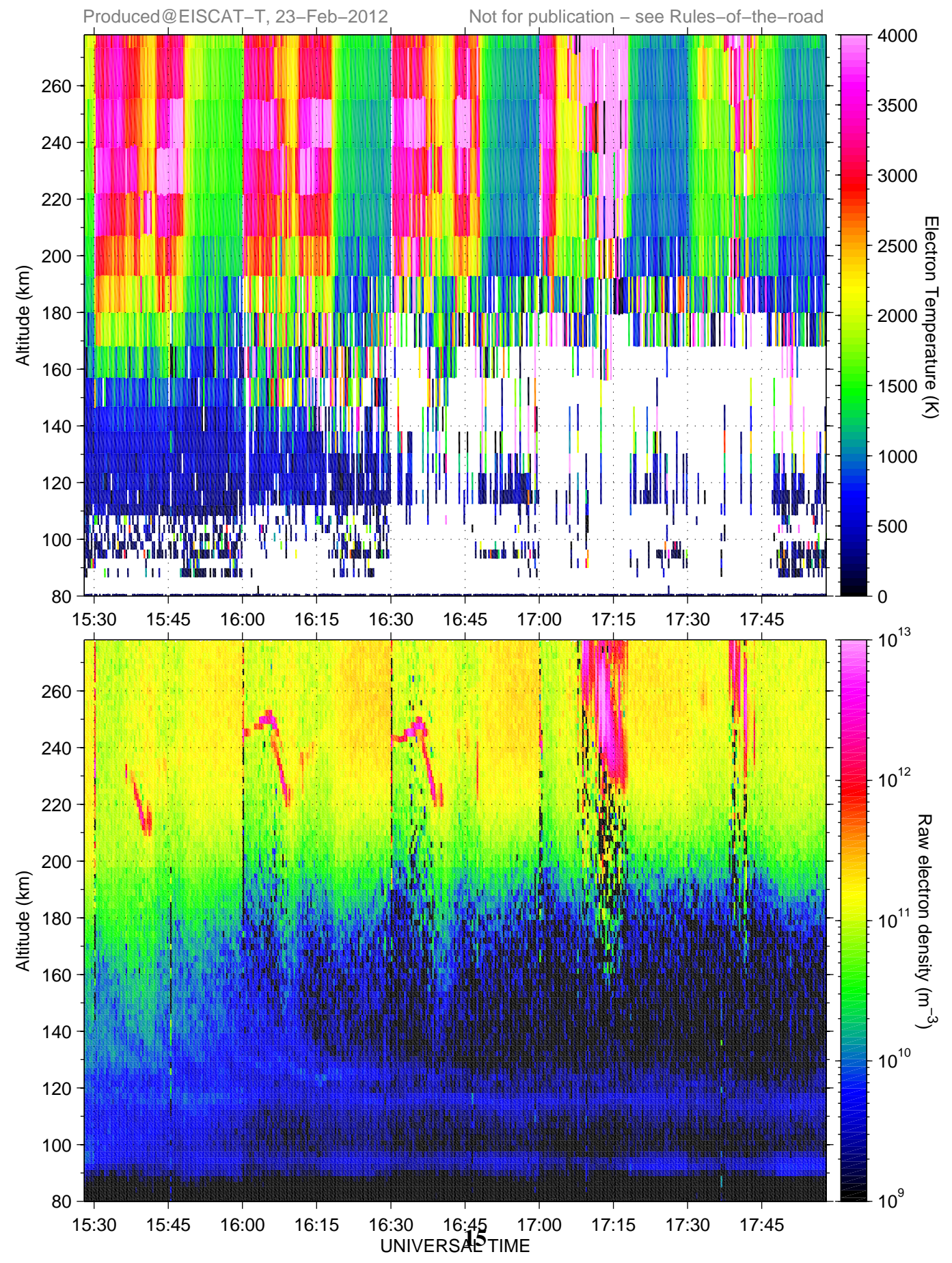



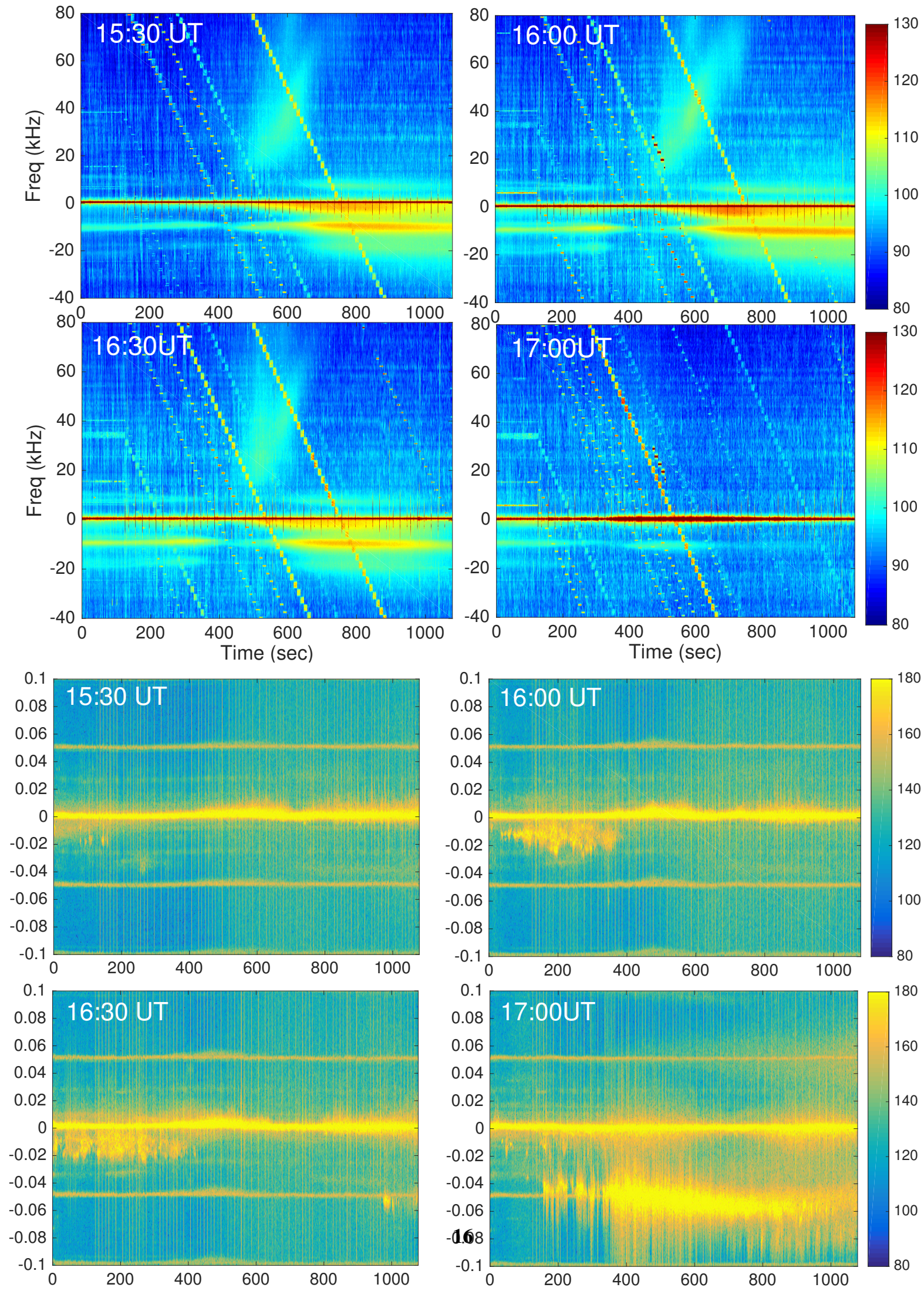